\title{
Noise Reduction in CT Images Using a Selective Mean Filter
}

\author{
Anam C. ${ }^{* * \odot}$, Adi K. ${ }^{1}$, Sutanto H. ${ }^{1}$, Arifin Z. ${ }^{2}$, Budi W. S. ${ }^{1}$, \\ Fujibuchi T.3. Dougherty G. ${ }^{4}$
}

\begin{abstract}
Background: Noise reduction is a method for reducing CT dose; however, it can reduce image quality.
\end{abstract}

Objective: This study aims to propose a selective mean filter (SMF) and evaluate its effectiveness for noise suppression in CT images.

Material and Methods: This experimental study proposed and implemented the new noise reduction algorithm. The proposed algorithm is based on a mean filter (MF), but the calculation of the mean pixel value using the neighboring pixels in a kernel selectively applied a threshold value based on the noise of the image. The SMF method was evaluated using images of phantoms. The dose reduction was estimated by comparing the image noise acquired with a lower dose after implementing the SMF method and the noise in the original image acquired with a higher dose. For comparison, the images were also filtered with an adaptive mean filter (AMF) and a bilateral filter (BF).

Results: The spatial resolution of the image filtered with the SMF was similar to the original images and the images filtered with the BF. While using the AMF, spatial resolution was significantly corrupted. The noise reduction achieved using the SMF was up to $75 \%$, while it was up to $50 \%$ using the BF.

Conclusion: SMF significantly reduces the noise and preserves the spatial resolution of the image. The noise reduction was more pronounced with $\mathrm{BF}$, and less pronounced with AMF.

Citation: Anam C, Adi K, Sutanto H, Arifin Z, Budi WS, Fujibuchi T, Dougherty G. Noise Reduction in CT Images Using a Selective Mean Filter. J Biomed Phys Eng. 2020;10(5):623-634. doi: 10.31661/jbpe.v0i0.2002-1072.

\section{Keywords}

X-Rays; Computed Tomography; Image Quality; Noise; Image Processing; Algorithms; Selective Mean Filter; Noise Reduction

\section{Introduction}

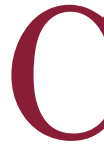

omputed tomography (CT) is an important imaging modality commonly used in clinical examinations [1,2]. Many studies have reported that the radiation dose during $\mathrm{CT}$ is greater than other imaging modalities [3-4]. This leads to a greater risk of cancer for the patient [5-7]. Efforts to reduce CT dose have been carried out by various methods, such as using iterative reconstruction (IR), tube current modulation (TCM), automatic exposure control (AEC), adjusting $\mathrm{kV}$ based on patient size, and so on [8-12]. One such procedure for reducing dose uses a noise reduction technique $[13,14]$. This procedure is carried out as follows: first, the images are acquired at a low input parameter (e.g.
${ }^{1} \mathrm{PhD}$, Department of Physics, Faculty of Sciences and Mathematics,

Diponegoro University,

Jl. Prof. Soedarto SH,

Tembalang, Semarang

50275, Central Java,

Indonesia

${ }^{2} \mathrm{MSc}$, Department of

Physics, Faculty of Sci-

ences and Mathematics,

Diponegoro University,

Jl. Prof. Soedarto SH,

Tembalang, Semarang

50275, Central Java,

Indonesia

${ }^{3} \mathrm{PhD}$, Department of

Health Sciences, Faculty

of Medical Sciences,

Kyushu University, 3-1-1

Maidashi, Higashi-ku,

Fukuoka 812-8582,

Japan

${ }^{4} \mathrm{PhD}$, Department of Ap-

plied Physics and Medi-

cal Imaging, California

State University Channel

Islands, Camarillo, CA

93012, USA

*Corresponding author: C. Anam

Department of Physics,

Faculty of Sciences and

Mathematics, Dipo-

negoro University, J.

Prof. Soedarto SH,

Tembalang, Semarang

50275, Central Java,

Indonesia

E-mail: anam@fisika.

fsm.undip.ac.id

Received: 13 February 2020

Accepted: 7 March 2020 
low $\mathrm{mA}$ or fast rotation time), resulting in low dose and noisy images, and second the noise is then reduced using a noise reduction filter [14]. The main problem of the noise reduction technique is a decrease in spatial resolution of the image [15]. The low-resolution image may potentially lead to misinterpretation when the diagnosis of diseases and abnormalities. For this reason, several efforts have been made to ensure that spatial resolution does not significantly decrease [16-24].

There are several algorithms for noise reduction, e.g. the mean filter (MF) [14], adaptive mean filter (AMF) [16, 17], and bilateral filter (BF) [18-22]. The simplest one is the MF [14], the AMF is a refinement of the mean filter $[16,17]$ and the BF is considered as a stateof-the-art noise reduction technique [22]. The $\mathrm{BF}$ combines filter range and filter domain in a specific window size. The BF is a noniterative adaptive smoothing filter reducing noise with maintaining the edges of the objects within an image [18]. The BF has three variables, including the window size, the filter range, and the filter domain. This allows the $\mathrm{BF}$ to maintain a high degree of fine detail and texture of image. However, the BF technique requires relatively heavy computation and is mathematically complex so that development of a simple and fast algorithm for noise reduction with excellent image quality would be very significant $[23,24]$. In this paper, we aim to propose and evaluate a novel noise reduction technique based on the MF with the aim of reducing the noise in an image without significantly reducing the spatial resolution.

\section{Material and Methods}

\section{The proposed algorithm}

This experimental study was carried out on the new noise reduction algorithm. Previous algorithms of noise reduction included mean filter (MF), adaptive mean filter (AMF), and bilateral filter $(\mathrm{BF})$. A MF simply replaces each pixel value in an image $(I(x, y))$ with the mean value of itself and its neighbors $(I(x+i, y+j))$ using equation (1).

$I_{M F}(x, y)=\frac{\sum_{i=-\frac{n-1}{2}, j=-\frac{m-1}{2}}^{+\frac{n-1}{2},+\frac{m-1}{2}} I(x+i, y+j)}{N}$

Where $N=n \times m$ and $n$ and $m$ are odd positive integer numbers. In this study, $n=m=$ 5. The mean filter is simple to implement, but significantly reduces the spatial resolution of the image. An adaptive mean filter (AMF) was introduced to reduce the noise based on the local variance of the image. The original image $(I(x, y))$ was filtered using equation $(2)$

$I_{A M F}(x, y)=I_{M F}(x, y)+\frac{\sigma_{L}^{2}-\sigma_{g}^{2}}{\sigma_{L}^{2}}\left(I(x, y)-I_{M F}(x, y)\right)(2)$

Where $\sigma_{g}$ is the global variance of the noisy image, and $\sigma_{L}$ is the local variance of the image. In relatively homogeneous areas, $\sigma_{L}$ is small and the filter performs more noise reduction so that the equation tends to the value of $I_{M F}(x, y)$, and in the area of the edges, $\sigma_{L}$ is large and the filter performs little noise reduction so that the equation tends to the value of $I(x, y)$. The result is an image with low noise, but the spatial resolution can still be significantly compromised $[16,17]$.

In order to improve spatial resolution in the resulting image, the BF was introduced. The $\mathrm{BF}$ incorporates the pixel value similarity (i.e. filter range) and the geometric range in an $n \times$ $m$ sliding window [18-22]. The BF was calculated using the equation (3).

$I_{B F}(x, y)=\frac{1}{k} \sum_{i=\frac{n-1}{2}, j=-\frac{m-1}{2}}^{+\frac{n-1}{2}+\frac{m-\frac{1}{2}}{2}} e^{-\frac{\|x-i\|^{2}+\|y-j\|^{2}}{2 \sigma_{d}^{2}}} e^{-\frac{(I(x, y)-I(x+i, y+j))^{2}}{2 \sigma_{r}^{2}}} I(x, y)$

Where $k$ is normalization constant and calculated by the equation (4)

$k=\sum_{i=-\frac{n-1}{2}, j=-\frac{m-1}{2}}^{+\frac{n-1}{2},+\frac{m-1}{2}} e^{-\frac{\|x-i\|^{2}+\|y-j\|^{2}}{2 \sigma_{d}^{2}}} e^{-\frac{(I(x, y)-I(x+i, y+j))^{2}}{2 \sigma_{r}^{2}}} I(x, y)$

Where $\sigma_{d}$ represents the geometric spread. 
By increasing $\sigma_{d}$, more neighboring pixels are utilized for denoising, resulting in increased filtering. In this study, $\sigma_{d}=2 . \sigma_{r}$ represents the pixel intensity spread. It should be carefully chosen and is usually determined by trial and error [22]. In this study, $\sigma_{r}$ was taken as the standard deviation of the image (SD).

We propose a new simple technique, which we refer to as a selective mean filter (SMF). Unlike the standard MF method which calculates the average pixel using all pixels in a particular kernel area, the SMF algorithm calculates the average value selectively. Pixel selection is characterized by a threshold value (h). If a neighboring pixel in a kernel is greater or smaller than the threshold value from the value of the central pixel then it is not considered as noise and is not included in the noise reduction process. Pixel selection is accomplished using equation (5)

$I^{\prime}(x+i, y+j)=\left\{\begin{array}{cll}I(x+i, y+j), & \text { if } & |I(x, y)-I(x+i, y+j)| \leq h \\ 0, & \text { if } & |I(x, y)-I(x+i, y+j)|>h\end{array}\right.$

For every $i$ and $j$, if $|I(x, y)-I(x+i, y+j)|>h$, then $N^{\prime}(x, y)=N-1$. The noise image reduction is then calculated using equation (6).

$$
I_{S M F}(x, y)=\frac{\sum_{i=-\frac{n-1}{2}, j=-\frac{m-1}{2}}^{+\frac{n-1}{2},+\frac{m-1}{2}} I^{\prime}(x+i, y+j)}{N^{\prime}(x, y)}
$$

With equations (5) and (6), in the edge areas, it is likely that the differences of all the neighboring pixel values from the central pixel value exceeds $h$. In that case, the pixel value $I_{S M F}(x, y)$ is equal to $I(x, y)$. Conversely, in homogeneous regions, it is likely that the differences of all neighboring pixel values from the central pixel value is smaller than $h$. In that case, the pixel value $I_{S M F}(x, y)$ is equal to $I_{M F}$ $(x, y)$.

The noise reduction process of the SMF method is shown in Figure 1. The mean pixel value at the central pixel in a position of $(x$, $y$ ) was not calculated from all the pixels in a particular square kernel (i.e. union of blue and red areas), but was calculated only from the blue area where the differences of pixel values from the value of the central pixel were lower than the threshold value. The pixels, which were the outside of blue area, and even those still in the kernel of interest with their different pixel values higher than threshold value, were not used in the calculation.

The threshold $(h)$ was based on the magnitude of the standard deviation (SD) of the pixel values within an image, which is an indication of the amount of noise [25]. In this study, we used a threshold of 3 SDs to cover most of the image noise. The SD was automatically calculated using a previously proposed algorithm [26]. This automatically chooses the minimum value in the standard deviation map (SDM).

$$
S D=\min (S D M)
$$

The SMF was expected to significantly reduce noise, without corrupting the spatial resolution. Because it was MF-based, the technique is computationally light and fast so that it is easier to implement in clinical images than the BF. We used MatLab (Mathworks) to

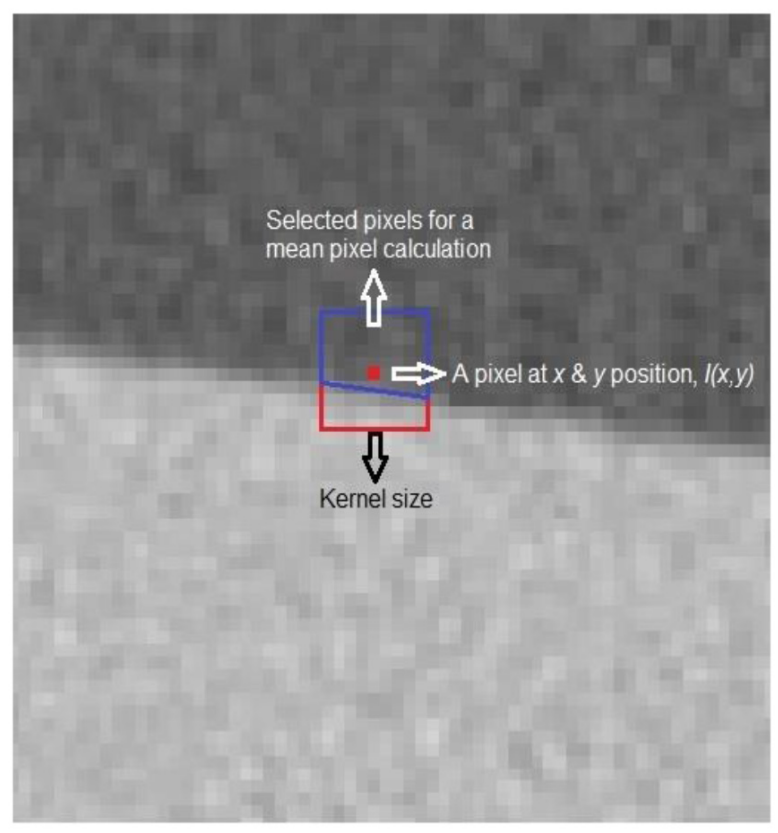

Figure 1: An illustration of selecting neighboring pixels for noise reduction in the selective mean filter (SMF) method. 
implement the algorithm.

\section{Evaluation of the proposed algo- rithm}

The evaluation of the proposed noise-reduction algorithm was carried out on images of 3 objects, viz. an in-house wire phantom, the AAPM CT performance phantom (model 610 , computerized imaging reference systems, Inc., Virginia, USA), and finally a pelvic anthropomorphic phantom (Kyoto Kagaku Ltd., Kyoto, Japan) (Figure 2). The images were filtered using our proposed method (SMF), and compared with the results of the AMF and the BF filters.

An in-house wire phantom was constructed from a $200 \mathrm{ml} \mathrm{CT}$ injector syringe (Kyorindo Nemoto Ltd., Japan) with a 4.8-cm diameter (Figure 2(a)). A tin wire with a $0.1-\mathrm{mm}$ diameter was positioned at the center of the syringe cylinder. The phantom was filled with water with 150-ml volume [27]. The in-house wire phantom was scanned by a CT Toshiba Alexion 4 installed at Kyushu University, with 120 $\mathrm{kVp}$ tube voltage, $150 \mathrm{~mA}$ tube current, $1 \mathrm{~s}$ rotation time, $7 \mathrm{~cm}$ field of view, $4 \mathrm{~mm}$ slice thickness, and a focal spot size of $1.1 \mathrm{~mm}$. The image was reconstructed with FC13 and FC30 reconstruction filters. The in-house wire phantom images were then filtered by the AMF, BF and SMF filters. The image spatial resolution was characterized using a modulation transfer function (MTF) curve. The MTF curves were calculated using an automated algorithm proposed previously [27]. Numerical values of $\mathrm{MTF}_{10}$ and $\mathrm{MTF}_{50}$ were obtained from all images. In addition, image noise was characterized using a noise power spectrum (NPS) curve, calculated using the ImQuest software [28]. Region of interests (ROIs) for NPS calculations was at the homogeneous region.

The resolution insert (Part Number 610-03) of the AAPM CT performance phantom is shown in Figure 2(b). The phantom consists of an acrylic equivalent object with eight sets of holes (five holes per set). The diameters of holes were $1.75,1.5,1.25,1.00,0.75,0.61$, 0.50 , and $0.40 \mathrm{~mm}$, respectively. The distance between each hole with same diameter is equal to the hole diameter. Each row is 5 $\mathrm{mm}$ apart. The phantom was scanned by a CT Siemens Emotion 6 installed at National Diponegoro Hospital (RSND), with $130 \mathrm{kVp}$, $23 \mathrm{~mA}, 600 \mathrm{~ms}$ time rotation, $10 \mathrm{~mm}$ slice thickness, $27 \mathrm{~cm}$ field of view, 0.85 pitch factor, and convolution kernel B41s. The spatial resolution was assessed by visual observation from the minimum hole diameter that could be distinguished. The noise of the images was determined from the SD of the homogeneous acrylic object.

The anthropomorphic phantom was scanned
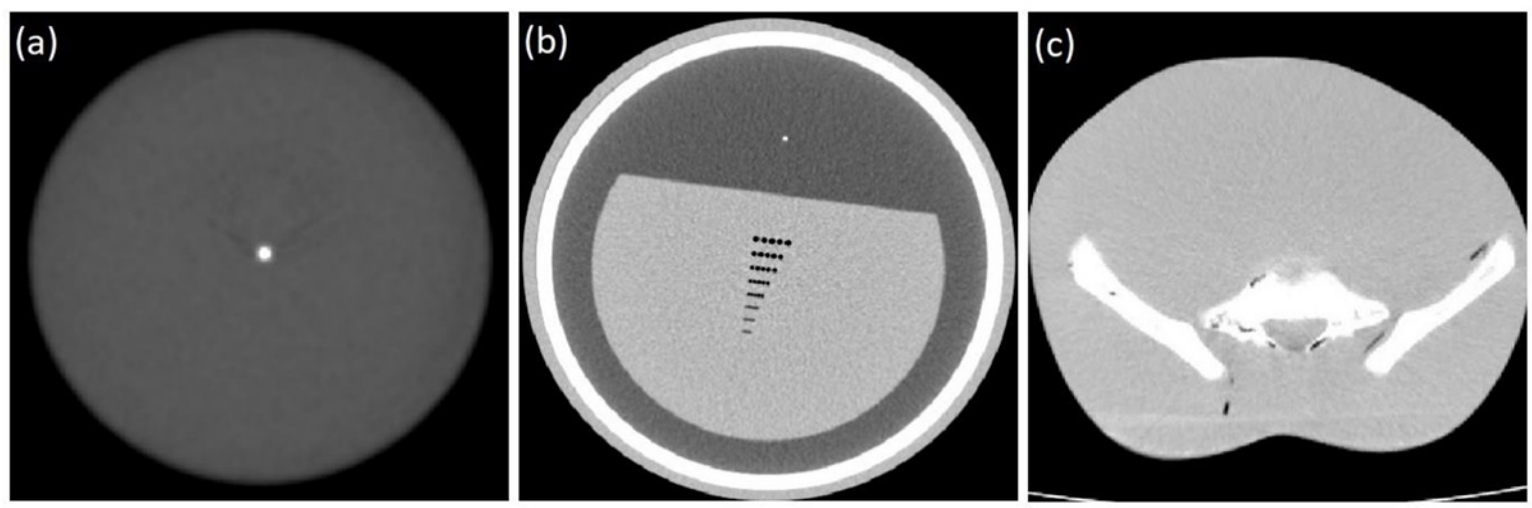

Figure 2: Images for evaluation of the proposed method of noise reduction. (a) In-house point phantom, (b) AAPM CT performance phantom, and (c) pelvis of anthropomorphic phantom. 
with a CT Toshiba Alexion 4 installed at $\mathrm{Ky}-$ ushu University, with $120 \mathrm{kVp}, 1 \mathrm{~s}$ time rotation, $7 \mathrm{~mm}$ slice thickness, $40 \mathrm{~cm}$ field of view, 1.5 pitch factor, and FC13 reconstruction filter. The anthropomorphic phantom was scanned three times with different three tube currents of 25, 50 and $100 \mathrm{mAs}$, corresponding to CTDIvol of 1.5, 3.0 and 6.0 mGys. The image of the anthropomorphic phantom in the pelvic part is shown in Figure 2(c). The noise value in the filtered images can be used to estimate the dose reduction achieved.

\section{Results}

The image of the in-house point phantom

The MTF curves of original and filtered images using $\mathrm{AMF}, \mathrm{BF}$ and $\mathrm{SMF}$ are shown in
Figure 3(a-b). It is clear that the MTFs of the filtered images using BF and SMF are very similar to the original unfiltered images. The MTF curves coinciding each other, indicating BF and SMF maintain the spatial resolution of the images. Conversely, images filtered with the AMF have poorer spatial resolution. The $\mathrm{MTF}_{50}$ and MTF 10 values are listed in Table 1. The NPS curves of original and filtered images using the AMF, BF and SMF are shown in Figure 3(c-d). For FC13, the NPS curve from SMF coincides with that from AMF, and the noise is significantly lower than the original image. The BF produces slightly more noise than the SMF. The same pattern is seen in the FC30. However, the difference in the NPS between the SMF and the BP is higher. These results reveal that $\mathrm{SMF}$ produces lower noise than $\mathrm{BF}$ with similar spatial resolution.
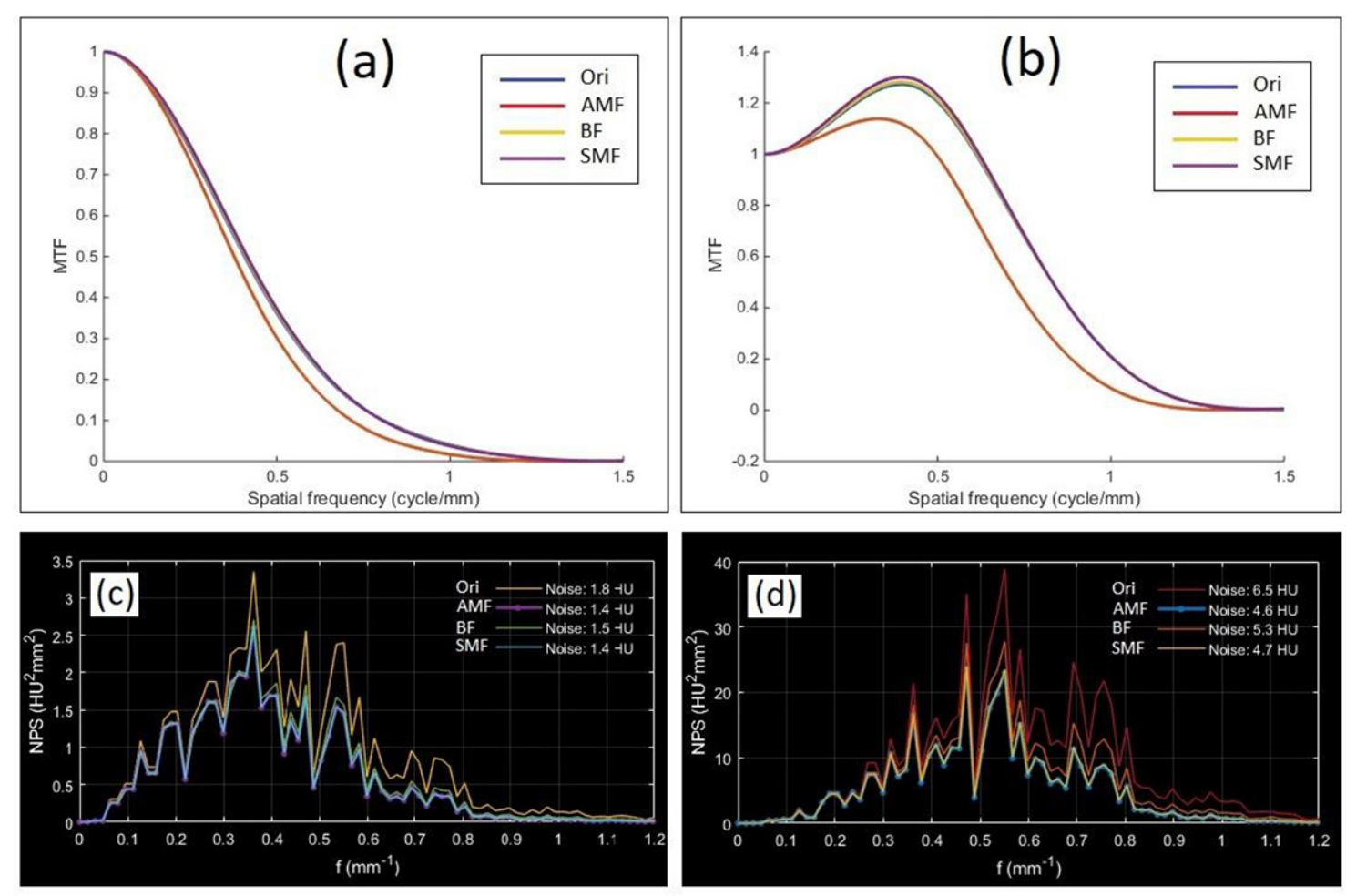

Figure 3: Modulation transfer function (MTF) and noise power spectrum (NPS) curves of original unfiltered images and filtered images using adaptive mean filter (AMF), bilateral filter (BF) and selective mean filter (SMF) for images reconstructed using FC13 and FC30. (a) MTF for FC13, (b) MTF for FC30, (c) noise power spectrum (NPS) for FC13, and (d) NPS for FC30. 
The image of AAPM CT phantom

The image of the spatial resolution module in the AAPM CT performance phantom is

Table 1: The modulation transfer function $\left(\mathrm{MTF}_{50}\right.$ and $\left.\mathrm{MTF}_{10}\right)$ for original and filtered images using adaptive mean filter (AMF), bilateral filter (BF) and selective mean filter (SMF).

\begin{tabular}{|c|c|c|c|c|}
\hline \multirow[t]{2}{*}{ Images } & \multicolumn{2}{|c|}{$\begin{array}{c}\text { MTF }_{10} \text { (cycles/ } \\
\mathrm{mm})\end{array}$} & \multicolumn{2}{|c|}{$\begin{array}{c}\text { MTF }_{50} \text { (cycles/ } \\
\mathrm{mm} \text { ) }\end{array}$} \\
\hline & FC13 & FC30 & FC13 & FC30 \\
\hline Original & 0.833 & 1.119 & 0.409 & 0.839 \\
\hline AMF & 0.733 & 1.016 & 0.380 & 0.722 \\
\hline $\mathrm{BF}$ & 0.832 & 1.120 & 0.412 & 0.839 \\
\hline SMF & 0.832 & 1.118 & 0.416 & 0.840 \\
\hline
\end{tabular}
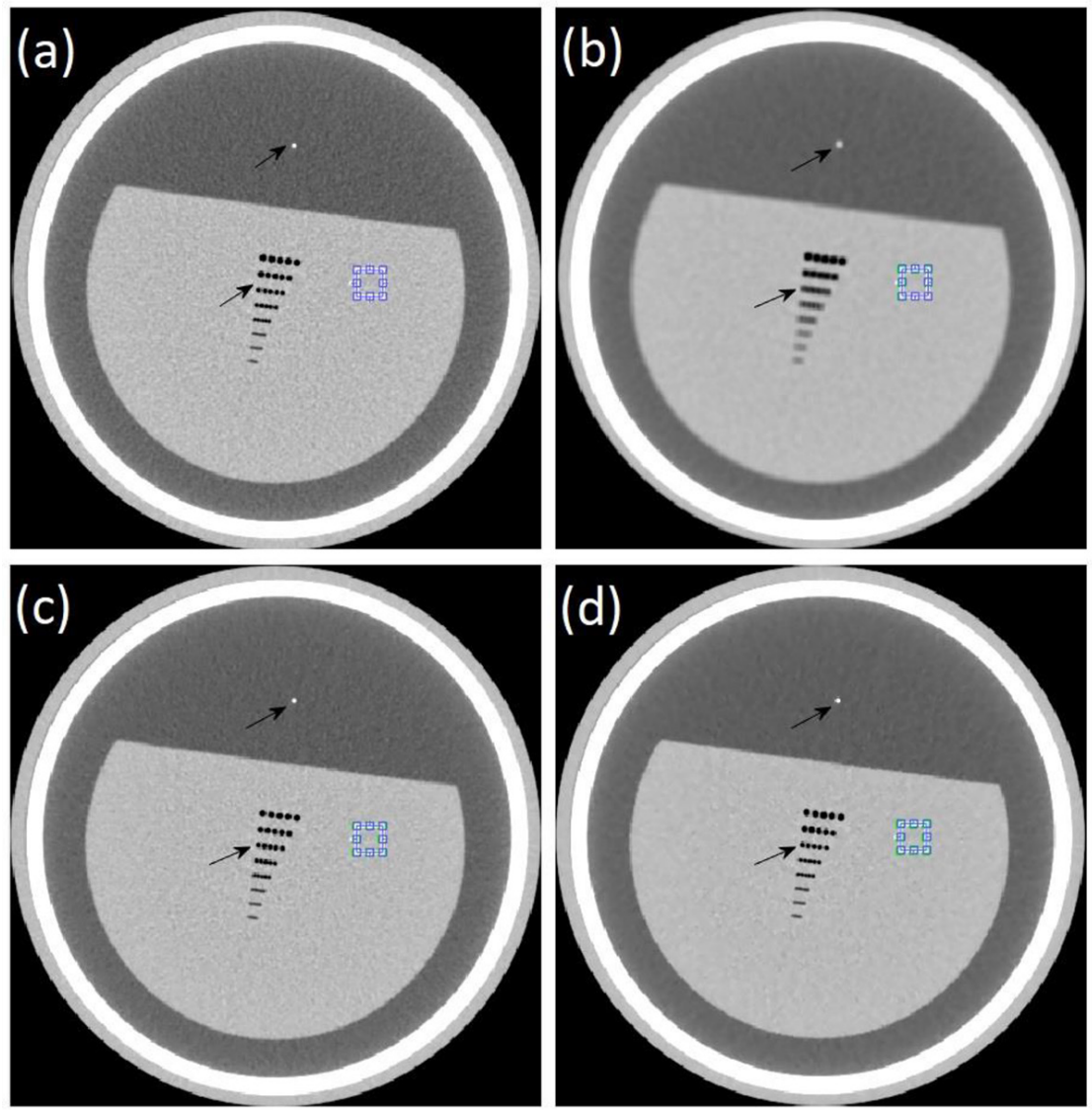

Figure 4: Image of spatial resolution module in the AAPM CT performance phantom. (a) Unfiltered original image, (b) Filtered image with the adaptive mean filter (AMF), (b) Filtered image with the bilateral filter (BF), and (d) Filtered image with the selective mean filter (SMF). 
respectively. It is clear that the noise using the SMF is smaller than that using the BF with comparable image spatial resolution.

\section{Dose reduction}

Figure 5 shows the anthropomorphic phantom image in the pelvic region scanned at a dose of $6.0 \mathrm{mGy}$, which was then filtered using the AMF, BF and SMF. The phantom was also scanned with half dose (3.0 mGy), and with a quarter the dose $(1.5 \mathrm{mGy})$, and noise reduction was again performed using the AMF, BF, and our proposed SMF. Noise for each image was calculated and the box-plots of noise are shown in Figure 6. The noise in the image was reduced by incremental dose as expected. However, applying the SMF to the im- ages scanned with doses of 1.5 and $3.0 \mathrm{mGy}$ reduced the noise to a level comparable to that obtained with a dose of $6.0 \mathrm{mGy}$, i.e. the SMF potentially reduces the dose required to obtain a certain noise level up to $75 \%$, without any significant reduction in spatial resolution. By comparison, the $\mathrm{BF}$ was only able to reduce the dose up to $50 \%$ from the dose of $3.0 \mathrm{mGy}$ to $1.5 \mathrm{mGy}$ for a similar noise level, and a $50 \%$ dose reduction was not achieved from the dose of $6.0 \mathrm{mGy}$ to $3.0 \mathrm{mGy}$.

\section{Discussion}

Noise reduction is one way to optimize the dose of $\mathrm{CT}$ scans, by reducing the radiation dose while maintaining image quality. Other methods include tube current modulation
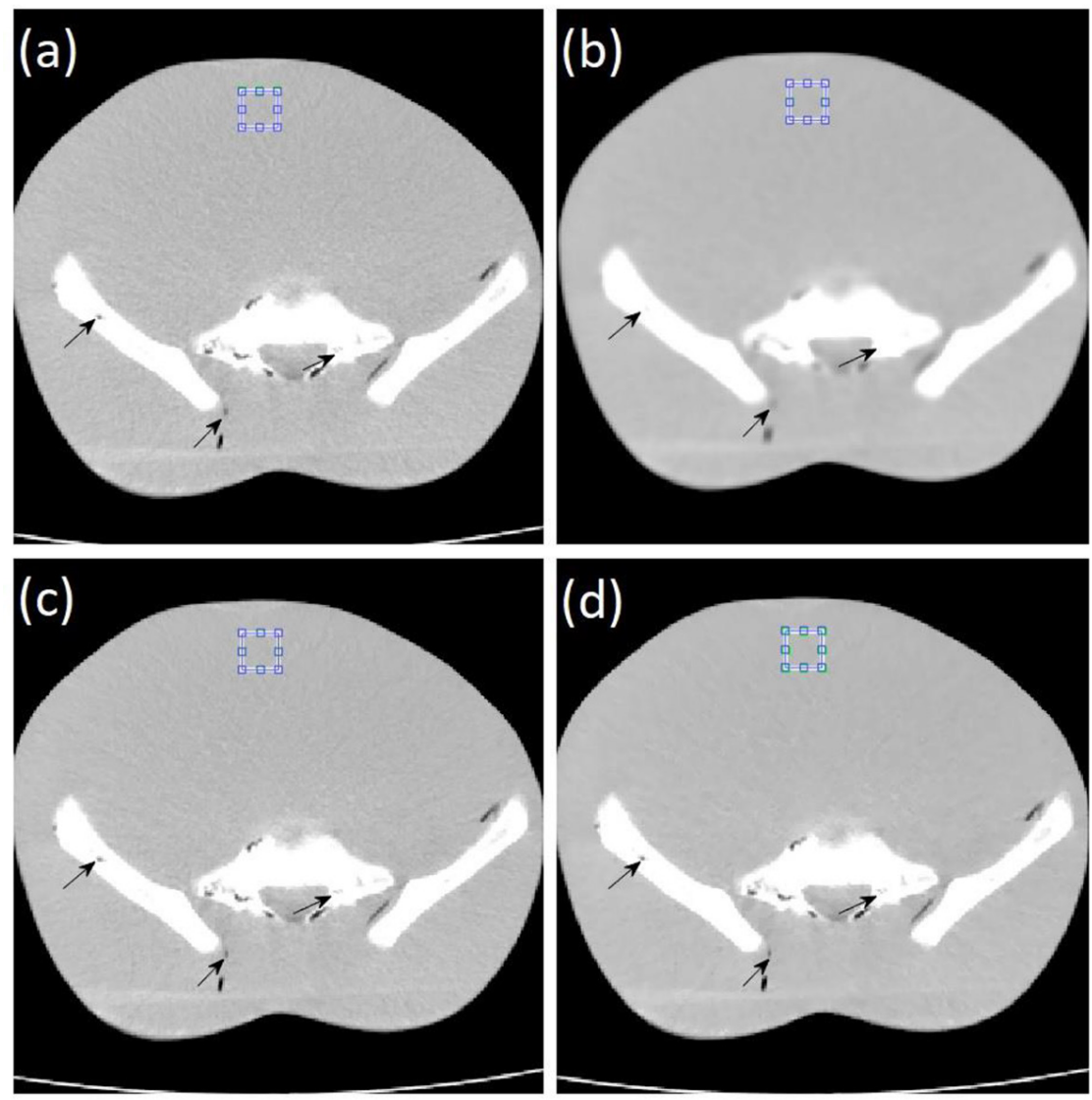

Figure 5: Image of anthropomorphic phantom in the pelvic region scanned at a dose of $6.0 \mathrm{mGy}$. (a) Original unfiltered image, (b) Adaptive mean filter (AMF) filtered image, (c) Bilateral filter (BF) filtered image, (d) filtered image with selective mean filter (SMF). There were 10 slices of every set of images for each radiation dose. 


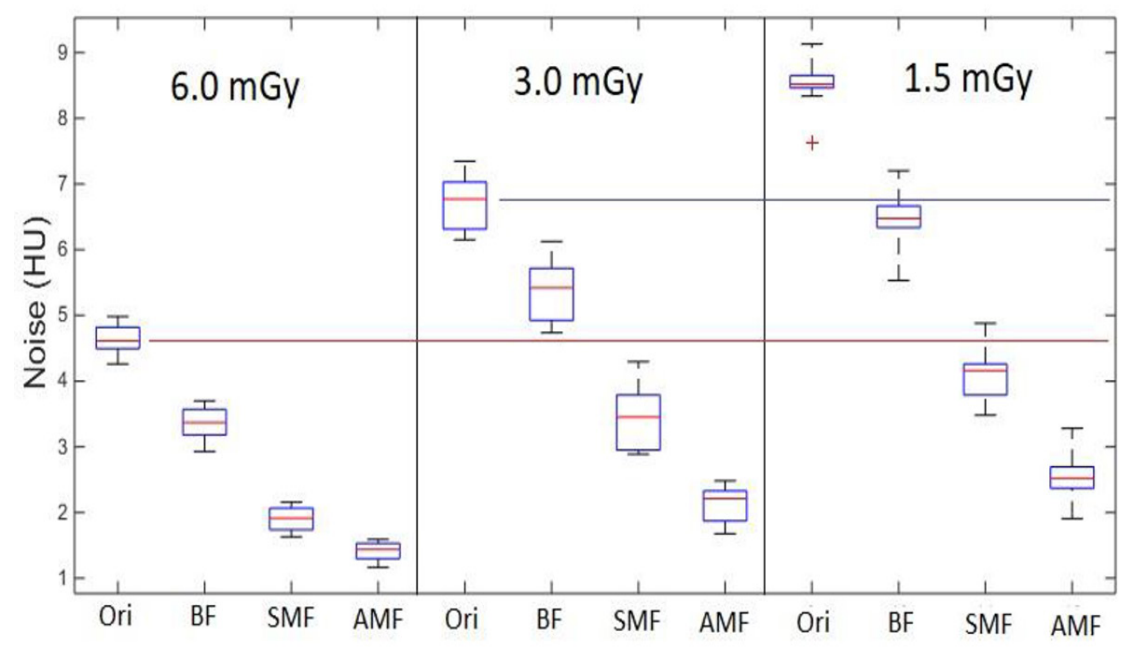

Figure 6: The box-plots of noise in the anthropomorphic phantom images obtained with doses of 6.0, 3.0, and $1.5 \mathrm{mGy}$, and with filtering using the adaptive mean filter (AMF), bilateral filter (BF), and selective mean filter (SMF).

(TCM) [29], iterative reconstruction (IR) [30], increased detector efficiency [31], and the use of an adaptive collimator [31]. With some of these techniques, it is possible to reduce the dose to less than $1 \mathrm{mSv}$ and still obtain an image quality level that can be used to make a diagnosis.

Noise reduction can be accomplished in both the projection space and the reconstructed image space. In the reconstructed image space, it can be implemented without access to the projection data, whereas it requires the projection data in projection space. The main drawback with the noise reduction method is the degradation of spatial resolution. Several proposals have been made to overcome this such as the adaptive mean filter (AMF) [16]. However, this study shows that with AMF, the spatial resolution drops significantly in general. Some researchers have combined the AMF approach with edge detection [17], i.e. the AMF is only carried out at the non-edge area, while in the edge area, filtering is not carried out. Although this approach can be beneficial, the image appears unnatural because boundaries appear in the edge areas [17].
Noise reduction techniques considered successful in maintaining the spatial resolution of the image, including the bilateral filter (BF) [32]. In the BF approach, noise reduction is carried out by convolving a Gaussian noise model with the pixels in the image and then incorporating the pixel values of the image. There are two main parameters, including determining the filter range and determining the domain filter. Some researchers reported that the BF was able to reduce doses by half while maintaining an image quality for diagnosis [20-22]. Even though in the BF filter, noise is modeled in a Gaussian distribution using the value of image standard deviation (SD), the $\mathrm{SD}$ values are not actually taken from the image. Al-Hinnawi reported that the optimum range of filter was between 0.05 and 0.5 pixels [22]. The current study points out that the BF is only able to reduce the dose by about $50 \%$ from a dose of $3 \mathrm{mGy}$ to $1.5 \mathrm{mGy}$, and less than $50 \%$ for a dose change from $6 \mathrm{mGy}$ to 3 mGy.

The SMF proposed in this study is computationally simpler than the BF since it uses a mean filter (MF)-based approach. The filtering 
process is carried out only by calculating the mean value of neighboring pixels and itself. However, the neighborhood value involved in the calculation is carried out selectively, i.e. the difference in value does not exceed a certain threshold value. This threshold value is based on the SD value of the image obtained directly from the image automatically. For practical considerations, the threshold value can be achieved by 3 SDs. From this study, it was found that the SMF could reduce the dose to $75 \%$ (from a dose $6.0 \mathrm{mGy}$ to $1.5 \mathrm{mGy}$ ), i.e. the SMF is a better filter than the BF, with comparable spatial resolution of the image, making it preferable for most applications. The SMF method is about $54 \%$ faster than the BF method. Using our netbook (Lenovo Ideapad 330S with intel Core i5), the computing time of the SMF method was $1.64 \pm 0.05 \mathrm{~s}$ and the BF method was $3.56 \pm 0.10 \mathrm{~s}$ for one slice of image.

The limitation of SMF (and also the BF) is that if the noise in the image is relatively high, the low-contrast object might be lost. If the noise is high, the pixel threshold in the SMF calculation is also high, and therefore the filtering is robustly executed. As a result, the edges of low-contrast objects, for example an object with contrast under $10 \mathrm{HU}$, will be corrupted. If these objects are small, they may be lost completely. This limitation is experienced by almost all existing filtering methods. An effectiveness test of the SMF on low-contrast objects has not been done yet. One way to increase the effectiveness of the SMF on low-contrast objects might do using statistical approach. The selection of pixels in the SMF would be determined by not only the threshold value $(h)$ but also a statistical test. However, incorporating a statistical selection of the pixels may lead to increased computation time.

The SD used in the current study is obtained automatically using an algorithm proposed previously by Anam et al, [26]. The SD is the minimum value of the SD map measured using an ROI of about $1 \mathrm{~cm}^{2}$. This SD value is about half the noise value obtained by Christianson et al., [33] using the global noise method, obtained as the highest frequency of the SD map. It can also be calculated manually in a homogeneous manner, but it may become less effective for the use in a clinical environment because of relatively long time.

The evaluation in this preliminary study was carried out on the in-house wire phantom, the AAPM CT performance phantom, and an anthropomorphic phantom. A more comprehensive study needs to be conducted by involving many clinical images with direct assessment from radiological experts and/or using more comprehensive physical parameters or comprehensive image texture methods such as correlation, uniformity, entropy, and homogeneity.

Another state-of-the-art filter is a non-local mean (NLM) filter [34-38]. The NLM exploits similarity between nearby image patches to estimate an image structure [38] and only employs the pixels within image patches that have a similarity to the patch of interest. Hence, it maintains a high degree of image resolution. A direct comparison of the SMF with the NLM filter should be carried out so that the advantages and disadvantages of this SMF filer can be fully understood.

\section{Conclusion}

Noise reduction with a selective mean filter method has been proposed and developed in this study. Evaluation of the filter has been carried out on images of phantoms, and the results show that noise can be significantly reduced, while maintaining the spatial resolution of the image. It was found that this method has the potential to reduce patient doses up to $75 \%$. Further evaluation of the patients' images with a medical expert and comparison to other state-of-the-art noise reduction needs to be done to quantify the decrease in radiation dose and the magnitude of the optimal threshold value for clinical images for various examinations with CT scan. 


\section{Acknowledgment}

This work was funded by the Riset Publikasi Internasional Bereputasi Tinggi (RPIBT), Diponegoro University (contract number: 329116/UN7.P4.3/PP/2019).

\section{Conflict of Interest}

None

\section{References}

1. Huda W, Nickoloff EL, Boone JM. Overview of patient dosimetry in diagnostic radiology in the USA for the past. Medical Physics. 2008;35(12):5713-28. doi: 10.1118/1.3013604. PubMed PMID: 19175129.

2. Anam C, Haryanto F, Widita R, Arif I, Dougherty G. Automated calculation of water-equivalent diameter (DW) based on AAPM task group 220. Journal of Applied Clinical Medical Physics. 2016;17(4):320-33. doi: 10.1120/jacmp. v17i4.6171. PubMed PMID: 27455491. PubMed PMCID: PMC5690059.

3. John A, Thomas J, Andrew N. CT dosimetry: Comparison of measurement techniquesand devices. radiographics. 2008;28(1):245-53. doi: 10.1148/rg.281075024. PubMed PMID: 18203941.

4. Mazrani W, McHugh K, Marsden PJ. The radiation burden of radiological investigations. Archives of Disease in Childhood. 2007;92(12):1127-31. doi: 10.1136/adc.2006.101782. PubMed PMID: 18032642. PubMed PMCID: PMC2066089.

5. Pearce MS, Salotti JA, Little MP, McHugh K, Lee C, Kim KP, Howe NL, Ronckers CM, Rajaraman P. Sir Craft AW, Parker L, Berrington De González A. Radiation exposure from CT scans in childhood and subsequent risk of leukaemia and brain tumours: a retrospective cohort study. Lancet. 2012;380(9840):499-505. doi: 10.1016/ S0140-6736(12)60815-0. PubMed PMID: 22681860. PubMed PMCID: PMC3418594.

6. Mathews JD, Forsythe AV, Brady Z, Butler MW, Goergen SK, Byrnes GB, Giles GG, Wallace AB, Anderson PR, Guiver TA, McGale P. Cancer risk in 680000 people exposed to computed tomography scans in childhood or adolescence: data linkage study of 11 million Australians. BMJ. 2013;346:f2360. doi: 10.1136/bmj.f2360. PubMed PMID: 23694687. PubMed PMCID: PMC3660619.
7. Huang WY, Muo CH, Lin CY, Jen YM, Yang $\mathrm{MH}$, Lin JC, Sung FC, Kao CH. Paediatric head CT scan and subsequent risk of malignancy and benign brain tumour: a nation-wide population-based cohort study. British Journal of Cancer. 2014;110(9):2354-60. doi: 10.1038/ bjc.2014.103. PubMed PMID: 24569470. PubMed PMCID: PMC4007220.

8. McCollough CH, Primak AN, Braun N, Kofler J, Yu $\mathrm{L}$, Christner J. Strategies for reducing radiation dose in CT. Radiologic Clinics. 2009;47(1):27-40. doi: 10.1016/j.rcl.2008.10.006. PubMed PMID: 19195532. PubMed PMCID: PMC2743386.

9. Greess H, Lutze J, Nömayr A, Wolf $H$, Hothorn T, Kalender WA, Bautz W. Dose reduction in subsecond multislice spiral CT examination of children by online tube current modulation. European Radiology. 2004;14(6):995-9. doi: 10.1007/ s00330-004-2301-9. PubMed PMID: 15052502.

10. Söderberg M, Gunnarsson M. Automatic exposure control in computed tomography-an evaluation of systems from different manufacturers. Acta Radiologica. 2010;51(6):625-34. doi: 10.3109/02841851003698206. PubMed PMID: 20429764.

11. Singh S, Kalra MK, Khawaja RD, Padole A, Pourjabbar S, Lira D, Shepard JA, Digumarthy SR. Radiation dose optimization and thoracic computed tomography. Radiologic Clinics. 2014;52(1):1-5. doi: 10.1016/j.rcl.2013.08.004. PubMed PMID: 24267707.

12. Paterson A, Frush DP. Dose reduction in paediatric MDCT: general principles. Clinical Radiology. 2007;62(6):507-17. doi: 10.1016/j. crad.2006.12.004. PubMed PMID: 17467387.

13. Kamezawa $H$, Arimura $H$, Shirieda K, Kameda $\mathrm{N}$, Ohki M. Feasibility of patient dose reduction based on various noise suppression filters for cone-beam computed tomography in an imageguided patient positioning system. Physics in Medicine \& Biology. 2016;61(9):3609. doi: 10.1088/0031-9155/61/9/3609. PubMed PMID: 27065312.

14. Kalra MK, Maher MM, Sahani DV, Blake MA, Hahn PF, Avinash GB, Toth TL, Halpern E, Saini S. Low-dose CT of the abdomen: evaluation of image improvement with use of noise reduction filters-pilot study. Radiology. 2003;228(1):2516. doi: 10.1148/radiol.2281020693. PubMed PMID: 12832586.

15. Anam C, Fujibuchi T, Budi WS, Haryanto F, Dough- 
erty G. An algorithm for automated modulation transfer function measurement using an edge of a PMMA phantom: Impact of field of view on spatial resolution of CT images. Journal of Applied Clinical Medical Physics. 2018;19(6):24452. doi: 10.1002/acm2.12476. PubMed PMID: 30338920. PubMed PMCID: PMC6236841.

16. Hilts $M$, Jirasek $A$. Adaptive mean filtering for noise reduction in CT polymer gel dosimetry. Medical Physics. 2008;35(1):344-55. doi: 10.1118/1.2818742. PubMed PMID: 18293589.

17. Anam C, Haryanto F, Widita R, Arif I. New noise reduction method for reducing CT scan dose: Combining Wiener filtering and edge detection algorithm. AIP Conference Proceedings. 2015;1677(1):040004. doi: 10.1063/1.4930648.

18. Tomasi $C$, Manduchi R. Bilateral filtering for gray and color images. Sixth international conference on computer vision (IEEE Cat. No. 98CH36271); Bombay, India: IEEE; 1998. p. 839-84.

19. Zhang M, Gunturk BK. Multiresolution bilateral filtering for image denoising. IEEE Transactions on Image Processing. 2008;17(12):2324-33. doi: 10.1109/TIP.2008.2006658. PubMed PMID: 19004705. PubMed PMCID: PMC2614560.

20. Dong G, Acton ST. On the convergence of bilateral filter for edge-preserving image smoothing. IEEE Signal Processing Letters. 2007;14(9):61720. doi: 10.1109/LSP.2007.894966.

21. Leng S, Rueda V, Mccollough C. TU-A-201B-02: 20-Fold dose reduction using a gradient adaptive bilateral filter: demonstration using in vivo animal perfusion CT. Medical Physics. 2010;37(6). doi:10.1118/1.3469176.

22. Al-Hinnawi AR, Daear M, Huwaijah S. Assessment of bilateral filter on 1/2-dose chest-pelvis CT views. Radiological physics and technology. 2013;6(2):385-98. doi: 10.1007/s12194-0130212-7. PubMed PMID: 23605697.

23. Won Kim C, Kim JH. Realistic simulation of reduced-dose CT with noise modeling and sinogram synthesis using DICOM CT images. Medical Physics. 2014;41(1):011901. doi: 10.1118/1.4830431. PubMed PMID: 24387509.

24. Blezek DJ, Li Z, Bartholmai BJ, Manduca A, Erickson BJ. Clinically feasible CT denoising using a GPU-based non-local means algorithm. Society for Imaging Informatics in Medicine, 2011 Annual Meeting; Washington: DC; 2011.

25. Anam C, Budi WS, Adi K, Sutanto H, Haryanto
F, Ali MH, Fujibuchi T, Dougherty G. Assessment of patient dose and noise level of clinical CT images: automated measurements. Journal of Radiological Protection. 2019;39(3):783-93. doi: 10.1088/1361-6498/ab23cc.

26. Anam C, Arif I, Haryanto F, Lestari FP, Widita R, Budi WS, Sutanto H, Adi K, Fujibuchi T, Dougherty $\mathrm{G}$. An improved method of automated noise measurement system in CT images. $J$ Biomed Phys Eng. 2020.

27. Anam C, Fujibuchi T, Haryanto F, Budi WS, Sutanto H, Adi K, Muhlisin Z, Dougherty G. Automated MTF measurement in CT images with a simple wire phantom. Polish Journal of Medical Physics and Engineering. 2019;25(3):179-87. doi: 10.2478/pjmpe-2019-0024.

28. Performance Evaluation of Computed Tomography Systems. TG233 Resources [Internet]. [cited 2019 November 23]. Available from: http:// deckard.mc.duke.edu/ samei/tg233.html.

29. Anam C, Haryanto F, Widita R, Arif I, Dougherty $\mathrm{G}$, McLean D. Volume computed tomography dose index (CTDIvol) and size-specific dose estimate (SSDE) for tube current modulation (TCM) in CT scanning. International Journal of Radiation Research. 2018;16(3):289-97. doi: 10.18869/acadpub.ijrr.16.3.289.

30. Suyudi I, Anam C, Sutanto H, Triadyaksa P, Fujibuchi T. Comparisons of hounsfield unit linearity between images reconstructed using an adaptive iterative dose reduction (AIDR) and a filter back-projection (FBP) techniques. Journal of Biomedical Physics \& Engineering. 2020;10(2):215-24. doi: 10.31661/jbpe. v0i0.1912-1013. PubMed PMID: 32337189. PubMed PMCID: PMC7166214.

31. Kalender WA. Dose in x-ray computed tomography. Physics in Medicine \& Biology. 2014;59(3):R129-50. doi: 10.1088/00319155/59/3/R129. PubMed PMID: 24434792.

32. Vijaya G, Vasudevan V. A novel noise reduction method using double bilateral filtering. European Journal of Scientific Research. 2010;46(3):p331.

33. Christianson 0, Winslow J, Frush DP, Samei E. Automated technique to measure noise in clinical CT examinations. AJR Am J Roentgenol. 2015;205(1):W93-9. doi: 10.2214/ AJR.14.13613. PubMed PMID: 26102424.

34. Buades A, Coll B, Morel JM. A review of image denoising algorithms, with a new one. Multiscale Modeling \& Simulation. 2005;4(2):490-530. doi: 
$10.1137 / 040616024$

35. Li Z, Yu L, Trzasko JD, Lake DS, Blezek DJ, Fletcher JG, McCollough CH, Manduca A. Adaptive nonlocal means filtering based on local noise level for CT denoising. Medical Physics. 2014;41(1):011908. doi: 10.1118/1.4851635. PubMed PMID: 24387516.

36. Zhang $H$, Zeng $D$, Zhang $H$, Wang J, Liang Z, Ma J. Applications of nonlocal means algorithm in low-dose X-ray CT image processing and reconstruction: A review. Medical Physics. 2017;44(3):1168-85. doi: 10.1002/mp.12097. PubMed PMID: 28303644. PubMed PMCID:
PMC5381744.

37. Coupé $P$, Yger $P$, Prima $S$, Hellier $P$, Kervrann $C$, Barillot C. An optimized blockwise nonlocal means denoising filter for 3-D magnetic resonance images. IEEE Transactions on Medical Imaging. 2008;27(4):425-41. doi: 10.1109/ TMI.2007.906087. PubMed PMID: 18390341. PubMed PMCID: PMC2881565.

38. Coupé $P$, Hellier $P$, Kervrann C, Barillot $C$. Nonlocal means-based speckle filtering for ultrasound images. IEEE Transactions on Image Processing. 2009;18(10):2221-9. doi: 10.1109/ TIP.2009.2024064. 